\title{
Candida Endocarditis in Patients with Candidemia: A Single-Center Experience of 14 Cases
}

\author{
Florian Hitzenbichler (D) - Tobias Joha • Michaela Simon • Jirka Grosse • \\ Karin Menhart • Dirk Hellwig • Daniele Camboni · Sabine Sag • \\ Can Martin Sag $\cdot$ Frank Hanses $\cdot$ Bernd Salzberger $\cdot$ Arno Mohr
}

Received: 16 December 2019/ Accepted: 18 September 2020/Published online: 9 October 2020

(C) The Author(s) 2020

\begin{abstract}
A retrospective, single-center analysis of 14 cases of Candida endocarditis (from 355 candidemia cases during the years 2012-2019) revealed a high in-hospital mortality ( $57.1 \%)$, a high proportion of healthcare-associated infections (13/14) and a high treatment preference for echinocandins. Transthoracic echocardiography and ${ }^{18}$ F-FDG PET/CT had a sensitivity of $54.5 \%$ and $57.1 \%$, respectively. Patients were older than previously described and most patients with
\end{abstract}

Handling Editor: Vishnu Chaturvedi.

Electronic supplementary material The online version of this article (https://doi.org/10.1007/s11046-020-00492-3) contains supplementary material, which is available to authorized users.

F. Hitzenbichler $(\bowtie) \cdot$ T. Joha $\cdot$ F. Hanses ·

B. Salzberger · A. Mohr

Department of Infection Prevention and Infectious

Diseases, University Hospital Regensburg, Franz-Josef-

Strauß-Allee 11, 93053 Regensburg, Germany

e-mail: florian.hitzenbichler@ukr.de

M. Simon

Institute of Clinical Microbiology and Hygiene,

University Hospital Regensburg, Franz-Josef-Strauß-

Allee 11, 93053 Regensburg, Germany

J. Grosse · K. Menhart · D. Hellwig

Department of Nuclear Medicine, University Hospital

Regensburg, Franz-Josef-Strauß-Allee 11,

93053 Regensburg, Germany
Candida endocarditis had persistent candidemia for $\geq 3$ days despite antifungal therapy.

Keywords Candida . Candidemia - Endocarditis .

${ }^{18} \mathrm{~F}-\mathrm{FDG}$ PET/CT $\cdot$ Echinocandins $\cdot$ Outcome

\section{Introduction}

An increase in candidemia and in Candida endocarditis is expected due to rising numbers of patients with immunosuppression and intravascular or intracardiac devices $[1,2]$.

Only $2 \%$ of endocarditis cases are of fungal origin, but mortality in these patients is high and diagnosis is

D. Camboni

Department of Cardiothoracic Surgery, University Hospital Regensburg, Franz-Josef-Strauß-Allee 11, 93053 Regensburg, Germany

S. Sag · C. M. Sag

Department of Internal Medicine II, University Hospital

Regensburg, Franz-Josef-Strauß-Allee 11,

93053 Regensburg, Germany 
complicated due to a low sensitivity of blood cultures (BC) for Candida spp. [2].

In this case series, we describe our experience with Candida endocarditis (CE) in patients who were treated at our hospital in a 14-year period.

\section{Patients and Methods}

Regensburg University Hospital is an 839-bed tertiary care academic teaching hospital in Germany.

All reports on BCs positive for Candida spp. between January 1, 2006 and December 31, 2019 were retrieved from the microbiology laboratory database and patients' charts were reviewed retrospectively by three of the authors (TJ, AM, FHi).

Patients were classified as having $\mathrm{CE}$, if one of the following two conditions applied:

1. Positive Candida culture or histopathological findings consistent with fungal endocarditis from a surgically resected heart valve.

2. Echocardiographic evidence of endocardial involvement (in patients with candidemia).

All identified cases were reviewed independently by two of the authors (AM, FHi) for plausibility (where all available data including discharge letters and discharge diagnosis were taken into account).

Patients were considered to have healthcare-acquired candidemia if at least one of the following conditions applied [3]:

1. First positive Candida $\mathrm{BC} \geq 48 \mathrm{~h}$ after admission to an acute care hospital.

2. Admission from a long-term care facility or rehabilitation hospital with candidemia.

3. Attendance of a dialysis clinic in the last 30 days before admission with candidemia.

Persistent candidemia was defined as a $\mathrm{BC}$ positive for the same Candida species on $\geq 3$ days $(72 \mathrm{~h}$ ) despite initiation of antifungal therapy, which was similar to a definition previously published [4].

Species differentiation and antifungal susceptibility testing of Candida isolates were performed according to local laboratory standards (detailed information in the Supplement.)

In some patients, ${ }^{18}$ F-FDG PET/CT (PET/CT) imaging was performed. (Detailed information on the PET/CT protocol performed at our hospital in the
Supplement.) All available PET/CT images were reviewed retrospectively by one of the authors (JG), who was aware that $\mathrm{CE}$ was diagnosed in these patients.

PET/CT was not available in all patients of our study since it is not routinely performed in patients with endocarditis at our hospital.

The analysis was approved by the local ethics committee (No. 18-1240-104, waiver due to the retrospective nature of the study).

\section{Results}

Between January 1, 2012 and December 31, 2019355 cases of candidemia were recorded at our hospital. 15 patients were judged to have $\mathrm{CE}$ according to our definition (4.2\%). Before 2012 no cases of CE could be documented at our hospital (years 2006-2011; $n=165$ cases of candidemia), so these years were excluded from our analysis.

The distribution of cases with CE and candidemia over time is shown in Fig. 1. Detailed information on the 14 included patients is listed in Table 1.

One (female) patient had to be excluded from our analysis due to missing data. The patient was only seen in our emergency department, where in transesophageal echocardiogram (TEE) the diagnosis of endocarditis was made (previous valve surgery) and Candida tropicalis was isolated in three different BC sets. However, the patient was transferred to another university hospital after TEE (and before BCs were available) and is therefore lost for follow-up.

\section{Clinical Characteristics}

Eight of 14 patients were female (57.1\%), the median age was 71 years (range 46-86 years). Median Charlson score was 4 (range 2-8).

A total of 13 episodes of candidemia were healthcare-acquired with a central venous catheter (CVC) being the most likely source of fungemia in ten patients and a port catheter in two other patients (No. $11 \& 14)$. Twelve patients had previous intravenous antibiotic therapy.

Median hospital stay was 39 days (range 33-130 days). 
Fig. 1 Cases of candidemia and Candida endocarditis over the time period 2012-2019. Before 2012 (2006-2011) no cases of Candida endocarditis could be identified retrospectively

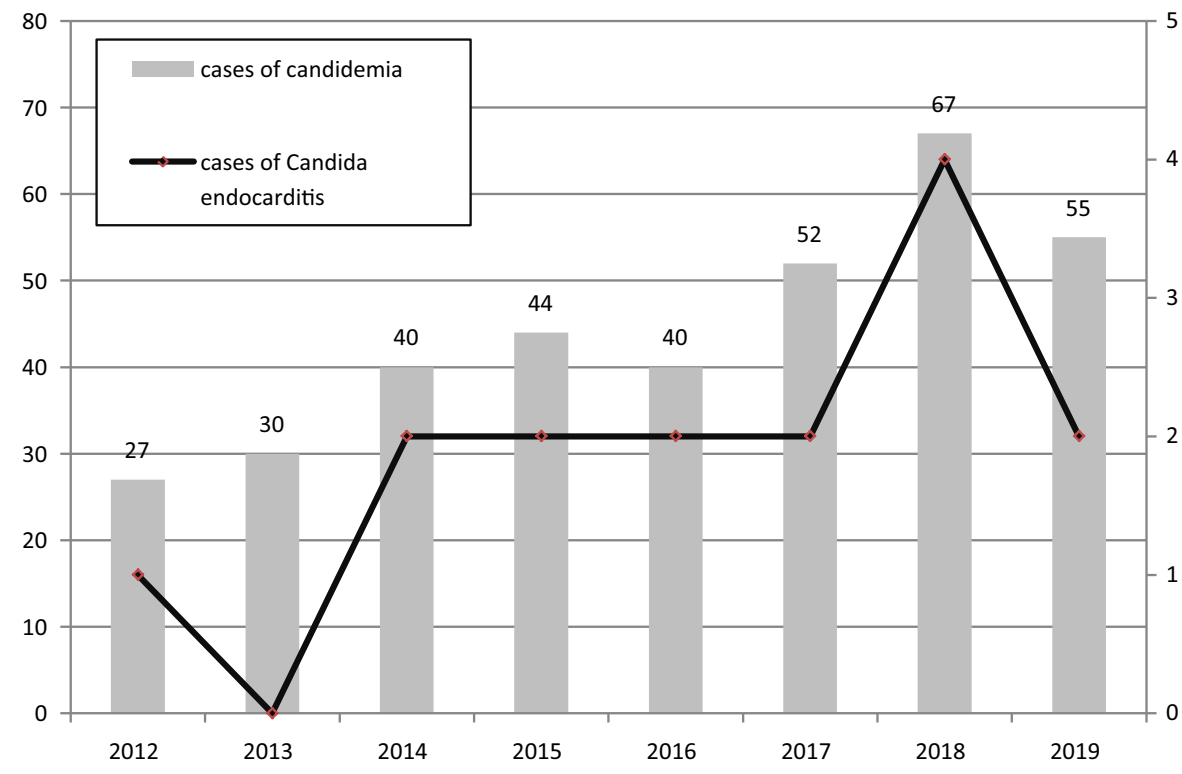

\section{Microbiology}

In twelve patients $C$. albicans was isolated. Other species (C. parapsilosis, C. glabrata) were found in only two patients (No. $10 \& 14$ ). Only two isolates were fluconazole resistant (C. albicans, patient No. 2, with previous fluconazole therapy; $C$. glabrata in patient No. 14 with intrinsic fluconazole resistance). No isolate was resistant to anidulafungin (one not tested, MIC range 0.02-0.06 $\mathrm{mg} / \mathrm{L}$ ).

Eleven patients $(78.6 \%)$ had persistent candidemia according to our definition.

\section{Diagnostics, Treatment and Outcome}

In $12 / 14$ patients an ophthalmological evaluation was available, involvement of the eye could be documented in only three patients (No. 2, 9, 14). Six patients had septic brain emboli (diagnosed in MRI); three patients had septic peripheral embolization (as diagnosed in CT scan and PET/CT). In 4/7 (57.1\%) patients, PET/CT confirmed endocarditis (PET/CT was available only in seven patients of our study).Vegetation size in TEE was $>10 \mathrm{~mm}$ in eight patients (median $12 \mathrm{~mm}$ ).

Infectious diseases consultation was requested in all patients at some point.

Echinocandins were used in all except one patient (No. 1) primarily. In six cases antifungal combination therapy was used, combining echinocandins with either liposomal amphotericin B or fluconazole. Three patients were switched from an echinocandin to liposomal amphotericin B due to various reasons (persistent candidemia, cerebral manifestation of CE).

Five patients received surgical treatment (valve replacement, removal of AICD), in all other cases operation was not judged feasible by the treating surgeon. Chronic suppressive therapy was discussed on a case-by-case basis and recommended in two cases. Median time interval between first candidemia episode and surgery was 9 days (range 4-20 days).

Eight patients died during their hospital stay (mortality rate: $57.1 \%$ ).

\section{Discussion}

$4.2 \%$ of patients with candidemia had CE-similar to a recent study where CE was diagnosed in $4.2 \%$ of patients with candidemia also [5]. Persistent candidemia was frequent $(84.6 \%)$ as was healthcareacquisition (92.3\%). Previous studies found a rate of healthcare-associated CE between 65 and $90 \%$ $[3,4,6,7]$.

The increase in CE rates over the years is surprising. However, it must be taken into account that in previous years guidelines on management of candidemia were different and echocardiography was not 


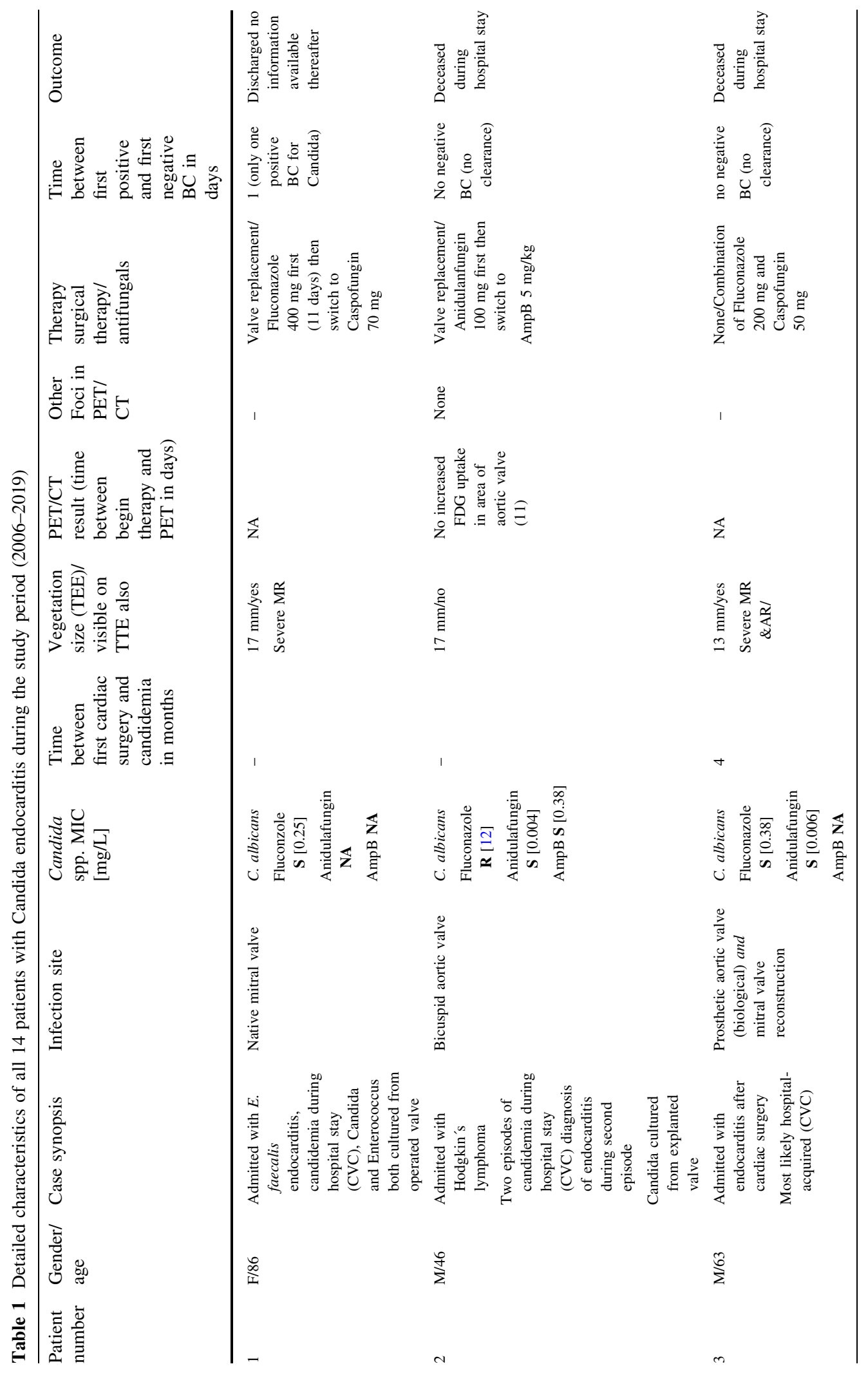




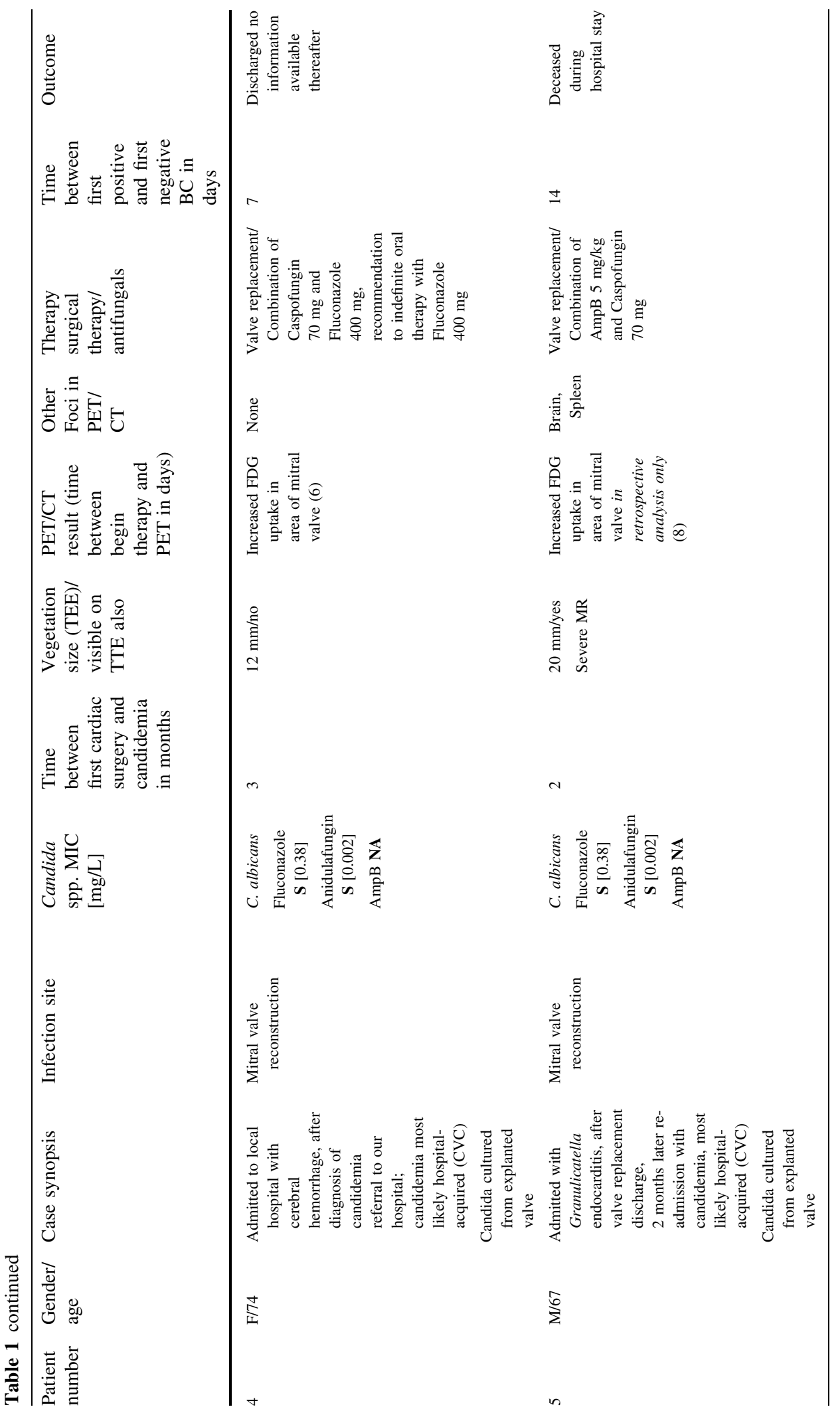




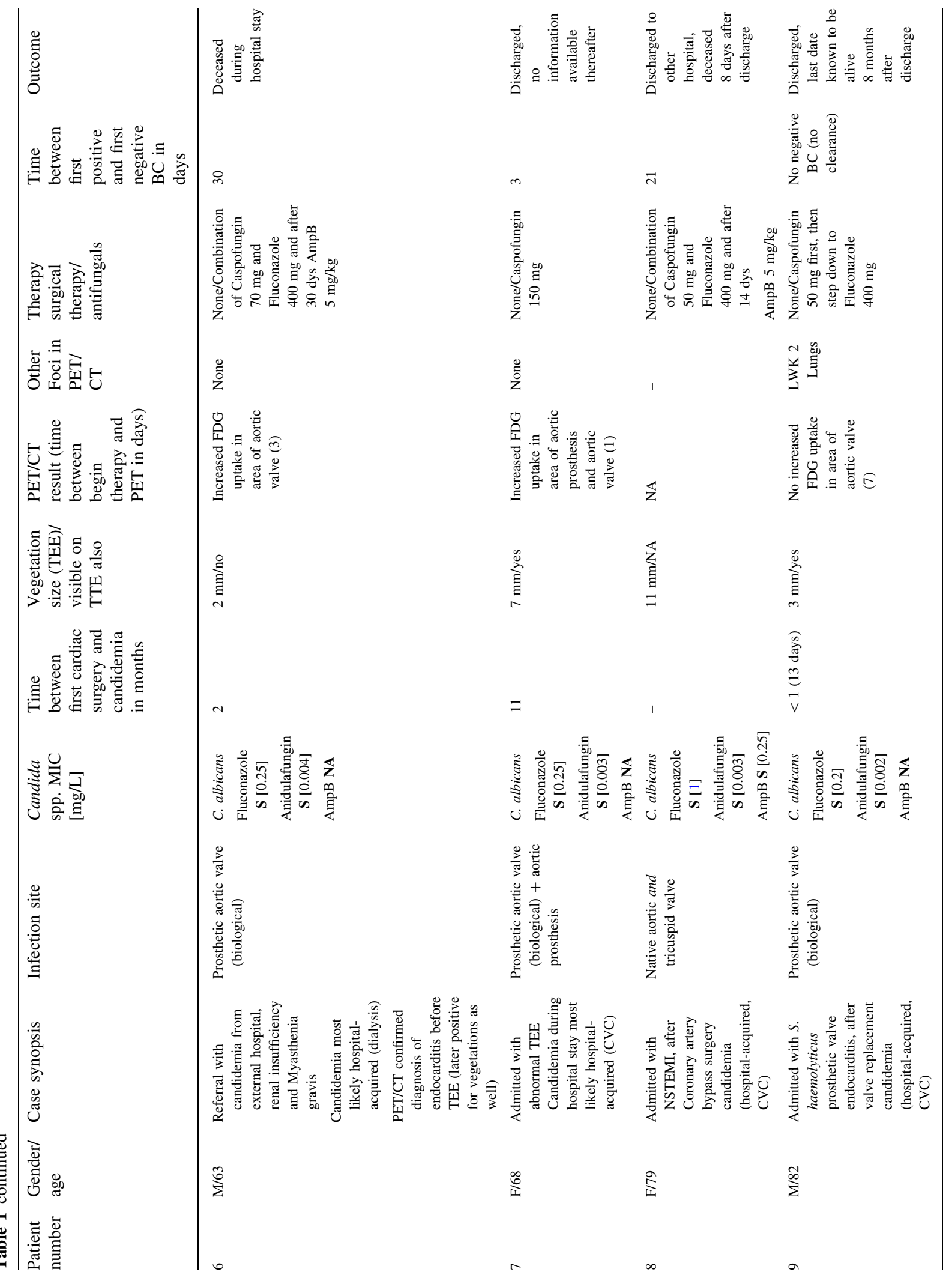




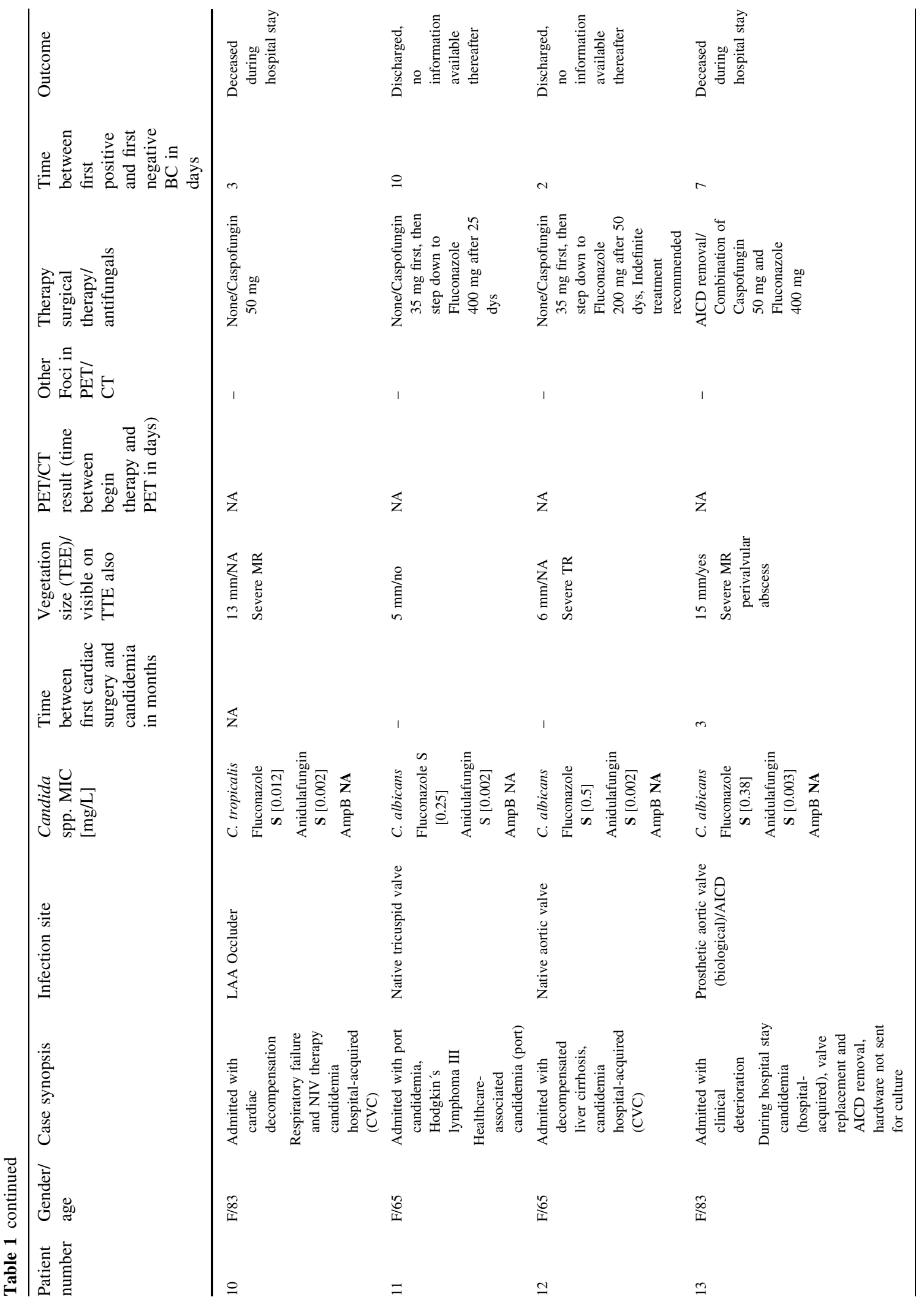




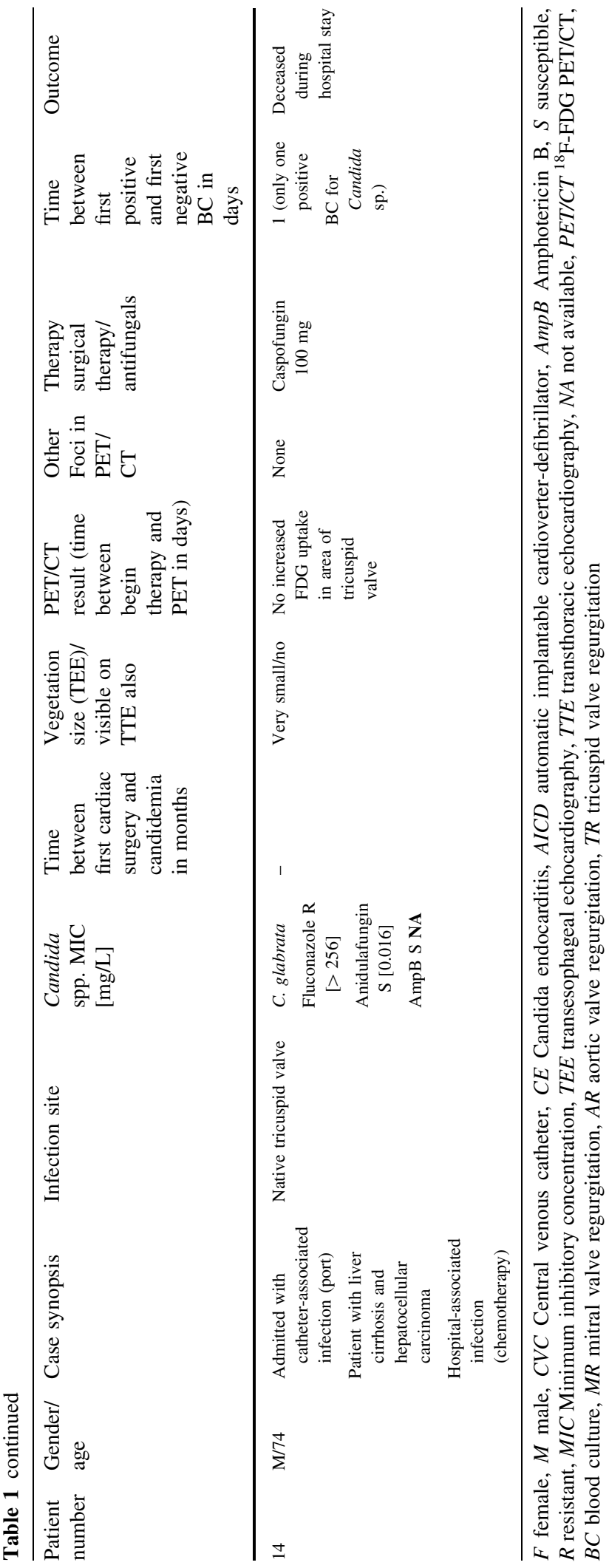

照 Springer 
routinely recommended. In a recent study, we could demonstrate an increase of candidemia cases in general over the last years (which might obviously lead to more cases of $\mathrm{CE}$ ). This might be due to an increase in sicker and older patients during the recent years at our hospital. Furthermore, TEE is performed more often in patients with candidemia at our hospital (in $11.6 \%$ of patients with candidemia in 2006-2008 vs. $40.3 \%$ in 2016-2018), which might also lead to a higher rate of diagnosed CE [8].

Only one of the patients described (No. 2) was younger than 60 years, all other patients were between 63 and 86 years old, which is different to other studies where the study population is slightly younger (median age: 55 years [7], 66 years [3], 59 years [2], mean age: 54 years [4]) In the pathological study of Vaideeswar et al. [6], patients were considerably younger (mean age: 23 years).

In $8 / 14$ patients, a history of cardiac surgery was present. Nearly all patients after prosthetic valve insertion or valve reconstruction developed CE within the first 6 months after surgery. In the study of Rivoisy and colleagues, the median time between surgery and CE was 8 months [3].

All prosthetic valves in our analysis were of biological origin. Similarly, Antinori et al. [2] found biological valves in all four patients with prosthetic valve $C E$. A recent meta-analysis observed $C E$ in both patients with biological $(n=31)$ and mechanical valves $(n=25)$ [1]. Patients with biological heart valves developed CE significantly earlier after surgery $(<1 \mathrm{a})$ than patients with mechanical valves. The authors speculated that formation of fungal biofilm requires some sort of biological component (either biological grafts or neoendocardium in mechanical valves) which might contribute to the time difference in development of $\mathrm{CE}$ after surgery (since endothelialisation of mechanical valves may take up to 24 months). Other studies did not differentiate between mechanical and biological prosthetic valves $[4,6,7]$.

Large heart valve vegetations were found on TEE in most patients (median size in this study: $12 \mathrm{~mm}$ ) and transthoracic echocardiogram (TTE) was not reliable in detecting vegetations (sensitivity $54.5 \%$ ). Large vegetations seem to be frequent in CE (in other studies: median size: $17 \mathrm{~mm}$ [3], $15 \mathrm{~mm}$ [7]).

PET/CT was not able to confirm CE in three patients despite of a large vegetation $(17 \mathrm{~mm})$ in one of them (No. 2). The same patient (No. 2), however, had leucopenia during the examination (leucocyte count $1.4 / \mathrm{nl}, 70 \%$ neutrophils). In recent studies, sensitivity of PET/CT was not compromised in patients with febrile neutropenia, but data on PET/ $\mathrm{CT}$ in patients with invasive fungal disease and neutropenia are scarce [9]. A French study reported $83 \%$ sensitivity of PET/CT in invasive candidiasis (liver, spleen, bone infections), but patients with $\mathrm{CE}$ were not included [10]. The low sensitivity compared to other studies that specifically evaluated the sensitivity of FDG PET/CT in endocarditis may also be due to the fact that patients in our study did not receive a PET/CT scan with the intention to diagnose endocarditis, but for the detection of an infectious focus in general. Thus, suppression of myocardial nuclide uptake, e.g., by a low-carbohydrate diet or intravenous preadministration of heparin, was not routinely performed.

To the best of our knowledge, this is the first series where sensitivity of PET/CT in CE was analyzed, but due to the small sample size, our results are still preliminary. Sensitivity is low in our cohort and comparable to TTE, however, the strength of PET/CT scans in patients with endocarditis might be in diagnosis of other foci or septic emboli or to confirm endocarditis in uncertain cases.

Echinocandins were used as first line therapy in $13 / 14$ patients in this analysis. Therapy was maintained if clearance of candidemia could be achieved; in all other cases combination therapy was used. Recent guidelines recommend either liposomal amphotericin B or higher-dosed echinocandins for CE [11].

In a retrospective analysis of 46 patients with prosthetic valve, CE patients receiving liposomal amphotericin B had a better outcome than those receiving monotherapy with an echinocandin [3]. In another recent study, patients with (both native and prosthetic valve) $\mathrm{CE}$ treated with an echinocandin had a similar outcome to those receiving liposomal amphotericin B despite a higher percentage of older patients and nosocomial infections in the echinocandin group [4]. In both studies, dosing regimens for echinocandins were not reported. The recent ESC guidelines for the management of infective endocarditis recommend to use echinocandins at higher doses also [12]. In only two patients of our study, higher doses of caspofungin were used, where no relevant 
side effects were noted. In some patients, higher doses of echinocandins were avoided due to comorbidities (like liver disease) or treatment was changed to combination therapy after infectious diseases consultation.

However, the role of combination therapy still remains unclear. Despite early use of echinocandins and a high rate of combination therapy, mortality in our series was $57 \%$. In two retrospective studies, a similar high mortality was seen [3, 7], other studies report a lower in-hospital mortality of below $40 \%$ $[1,4]$.

This study has the following limitations:

1. Since strict criteria for diagnosis of CE were applied, this might underestimate the true incidence of $\mathrm{CE}$ in this cohort, which might even be higher, since TEE is not always reliable for diagnosis of endocarditis. However, we tried to accommodate this problem by doublechecking for the diagnosis of endocarditis in the discharge letters. We were, however, not able to correlate our cohort with all patients with culture-negative endocarditis in the same period, since we were not able to retrieve all these patients from our data base. The same applies for patients with persistent candidemia (and without diagnosis of $\mathrm{CE}$ in discharge letters).

2. Standard incubation time of BCs was 5 days (supplement). This might underestimate incidence of $\mathrm{CE}$, since Candida diagnostic in $\mathrm{BC}$ is known to be limited with decreases sensitivity [11].

3. We were not able to provide drug levels of antifungals in our manuscript, since therapeutic drug monitoring (TDM) for fluconazole, echinocandins or liposomal amphotericin B is not available at our hospital.

In summary, Candida endocarditis is a rare condition (occurring in about $4 \%$ of patients with candidemia in our recent series) and cases are frequently healthcare-acquired. Risk factors include older age ( $>60$ years) and immunosuppression. Nearly all patients with $\mathrm{CE}$ had persistent candidemia. CE affected both native and prosthetic valves and should be considered an early event (mostly during the first 6 months) after biological heart valve surgery or valve reconstruction. Both PET/CT and TTE had a low sensitivity in diagnosis of CE $(<60 \%)$. The optimal treatment is still a matter of debate, but echinocandins are being more frequently used as first line therapy (maybe due to better tolerability). Mortality is still alarmingly high (clearly above 50\%).

Author contributions $\mathrm{FHi}, \mathrm{AM}$, BS and $\mathrm{FH}$ were involved in the conception and design of the study. TJ, AM, FHi, MS, JG, $\mathrm{KM}, \mathrm{SaS}, \mathrm{CMS}$ and DC contributed to data collection or data generation. FHi, AM, BS, DH, FH, JG, DC, CMS contributed to data analysis or data interpretation. FHi wrote the first draft of the manuscript and all authors reviewed and approved the final manuscript.

Funding Open Access funding enabled and organized by Projekt DEAL.

Data availability Data not publically available.

\section{Compliance with ethical standards}

Conflict of interest FHi.: Travel grants from Gilead Sciences, Lecture fees from MSD. A.M.: Travel grants from Gilead Sciences. B.S.: Educational grants from Gilead Sciences. M.S.: Lecture Fee from Beckton Dickinson. D.H.: Grants from Siemens and GE Healthcare, patent "Imaging Technologies" pending. J.G.: Grants and non-financial support from Bayer Healthcare. F.H.: Speaker and travel grants from Correvio, other grants (clinical study reimbursement) from Basilea and Portola. The other authors declare that they have no conflict of interest.

Open Access This article is licensed under a Creative Commons Attribution 4.0 International License, which permits use, sharing, adaptation, distribution and reproduction in any medium or format, as long as you give appropriate credit to the original author(s) and the source, provide a link to the Creative Commons licence, and indicate if changes were made. The images or other third party material in this article are included in the article's Creative Commons licence, unless indicated otherwise in a credit line to the material. If material is not included in the article's Creative Commons licence and your intended use is not permitted by statutory regulation or exceeds the permitted use, you will need to obtain permission directly from the copyright holder. To view a copy of this licence, visit http://creativecommons.org/licenses/by/4.0/.

\section{References}

1. Giuliano S, Guastalegname M, Russo A, Falcone M, Ravasio V, Rizzi M, et al. Candida endocarditis: systematic literature review from 1997 to 2014 and analysis of 29 cases from the Italian Study of Endocarditis. Expert Rev Anti Infect Ther. 2017;15:807-18.

2. Antinori S, Ferraris L, Orlando G, Tocalli L, Ricaboni D, Corbellino M, et al. Fungal endocarditis observed over an 8 -year period and a review of the literature. Mycopathologia. 2014;178:37-51. 
3. Rivoisy C, Vena A, Schaeffer L, Charlier C, Fontanet A, Delahaye F, et al. Prosthetic valve Candida spp. endocarditis: new insights into long-term prognosis-the ESCAPE study. Clin Infect Dis. 2018;66:825-32.

4. Arnold CJ, Johnson M, Bayer AS, Bradley S, Giannitsioti E, Miró JM, et al. Candida infective endocarditis: an observational cohort study with a focus on therapy. Antimicrob Agents Chemother. 2015;59:2365-73.

5. Fernández-Cruz A, Cruz Menárguez M, Muñoz P, Pedromingo M, Peláez T, Solís J, et al. The search for endocarditis in patients with candidemia: a systematic recommendation for echocardiography? A prospective cohort. Eur J Clin Microbiol Infect Dis. 2015;34:1543-9.

6. Vaideeswar P. Candidial endocarditis: a single-institute pathological analysis. Mycopathologia. 2015;180:81-7.

7. Siciliano RF, Gualandro DM, Sejas ONE, Ignoto BG, Caramelli B, Mansur AJ, et al. Outcomes in patients with fungal endocarditis: a multicenter observational cohort study. Int J Infect Dis. 2018;77:48-52.

8. Mohr A, Simon M, Joha T, Hanses F, Salzberger B, Hitzenbichler F. Epidemiology of candidemia and impact of infectious disease consultation on survival and care. Infection. 2020;48:275-84.
9. Vos FJ, Bleeker-Rovers CP, Oyen WJG. The use of FDG$\mathrm{PET} / \mathrm{CT}$ in patients with febrile neutropenia. Semin Nucl Med. 2013;43:340-8.

10. Leroy-Freschini B, Treglia G, Argemi X, Bund C, Kessler $\mathrm{R}$, Herbrecht R, et al. 18F-FDG PET/CT for invasive fungal infection in immunocompromised patients. QJM Int J Med. 2018;111:613-22.

11. Pappas PG, Kauffman CA, Andes DR, Clancy CJ, Marr KA, Ostrosky-Zeichner L, et al. Clinical practice guideline for the management of candidiasis: 2016 update by the Infectious Diseases Society of America. Clin Infect Dis. 2015;62:civ933.

12. Habib G, Lancellotti P, Antunes MJ, Bongiorni MG, Casalta J-P, Del Zotti F, et al. 2015 ESC Guidelines for the management of infective endocarditis. Eur Heart J. 2015;36:3075-128.

Publisher's Note Springer Nature remains neutral with regard to jurisdictional claims in published maps and institutional affiliations. 